\title{
Characterization of the structural determinants required for potent mechanism-based inhibition of human cytochrome P450 1A1 by cannabidiol
}

Satoshi Yamaori ${ }^{\mathrm{a}, 1}$, Yoshimi Okushima ${ }^{\text {a }}$, Ikuo Yamamoto ${ }^{\mathrm{b}}$, Kazuhito Watanabe a,c,*

a Department of Hygienic Chemistry, Faculty of Pharmaceutical Sciences, Hokuriku University, Ho-3 Kanagawa-machi, Kanazawa 920-1181, Japan

b School of Pharmaceutical Sciences, Kyushu University of Health and Welfare, 1714-1 Yoshino-machi, Nobeoka 882-8508, Japan

${ }^{\mathrm{c}}$ Organization for Frontier Research in Preventive Pharmaceutical Sciences, Hokuriku University, Ho-3 Kanagawa-machi, Kanazawa 920-1181, Japan

*Correspondence to:

Kazuhito Watanabe, Ph.D.

Department of Hygienic Chemistry

Faculty of Pharmaceutical Sciences

Hokuriku University

Ho-3 Kanagawa-machi, Kanazawa 920-1181, Japan

Tel: +81 76-229-6220, Fax: +81 76-229-6220

E-mail: k-watanabe@hokuriku-u.ac.jp

Abbreviations: CBD, cannabidiol; CBDHQ, CBD-hydroxyquinone; CBDM, CBD-2 -monomethyl ether; CBDD, CBD-2,6 -dimethyl ether; CBDV, cannabidivarin; CYP, cytochrome P450; EROD, 7-ethoxyresorufin $O$-deethylase. 


\begin{abstract}
We previously demonstrated that cannabidiol (CBD) was a potent mechanism-based inhibitor of human cytochrome P450 1A1 (CYP1A1). However, the moiety of CBD that contributes to the potent mechanism-based inhibition of human CYP1A1 remains unknown. Thus, the effects of compounds structurally related to CBD on CYP1A1 activity were examined with recombinant human CYP1A1 in order to characterize the structural requirements for potent inactivation by $\mathrm{CBD}$. When preincubated in the presence of NADPH for $20 \mathrm{~min}$, olivetol, which corresponds to the pentylresorcinol moiety of CBD, enhanced the inhibition of the 7-ethoxyresorufin $O$-deethylase activity of CYP1A1. In contrast, $d$-limonene, which corresponds to the terpene moiety of CBD, failed to inhibit CYP1A1 activity in a metabolism-dependent manner. Pentylbenzene, which lacks two free phenolic hydroxyl groups, also did not enhance CYP1A1 inhibition. On the other hand, preincubation of the CBD-2 -monomethyl ether (CBDM) and CBD-2,6-dimethyl ether (CBDD) enhanced the inhibition of CYP1A1 activity. Inhibition by cannabidivarin (CBDV), which possessed a propyl side chain, was strongly potentiated by its preincubation. Orcinol, which has a methyl group, augmented CYP1A1 inhibition, whereas its derivative without an alkyl side chain, resorcinol, did not exhibit any metabolism-dependent inhibition. The preincubation of CBD-hydroxyquinone did not markedly enhance CYP1A1 inhibition. We further confirmed that olivetol, CBDM, CBDD, CBDV, and orcinol, as well as $\mathrm{CBD}\left(k_{\text {inact }}=0.215 \mathrm{~min}^{-1}\right)$, inactivated CYP1A1 activity; their $k_{\text {inact }}$ values were $0.154,0.0638,0.0643,0.226$, and 0.0353 $\min ^{-1}$, respectively. These results suggest that the methylresorcinol structure in CBD may have structurally important roles in the inactivation of CYP1A1.
\end{abstract}

Keywords: cannabidiol; CYP1A1; inactivation; mechanism-based inhibition; structural requirement. 


\section{Introduction}

Cannabidiol (CBD) is one of the major constituents in marijuana [1]. Although another one of the major phytocannabinoids, $\Delta^{9}$-tetrahydrocannabinol, is psychoactive, CBD is not. It has been shown to exhibit several pharmacological effects such as prolonging drug-induced sleep as well as antiepileptic, anxiolytic, antiemetic, and anti-tumorigenic actions $[2,3]$. Some of these effects may be of therapeutic importance. Sativex ${ }^{\circledR}$, a medicine made from marijuana extracts containing CBD, has been clinically used for the symptomatic relief of neuropathic pain in patients with multiple sclerosis.

CBD is also known to inhibit CYP-mediated drug metabolism. The administration of CBD has been shown to reduce the systemic clearance of hexobarbital, which is metabolized by cytochrome P450 2C9 (CYP2C9), in humans [4]. We more recently demonstrated that CBD inhibited the catalytic activities of CYP1A1, CYP1A2, CYP2B6, CYP2C9, CYP2C19, CYP2D6, CYP3A4, and CYP3A5 in human liver microsomes and/or recombinant CYP enzymes [5-10]. We also showed that CBD more potently inhibited the activity of human CYP1A1 than the other CYP enzymes examined so far [5]. Furthermore, CBD had competitive and mechanism-based inhibitory effects on human CYP1A1 [5].

CBD contains pentylresorcinol and terpene moieties and is rotatable between their moieties (Fig. 1). We previously elucidated the structural requirements for the inhibition of cyclooxygenase, lipoxygenase, and CYP by CBD and its derivatives [6-8, 10-12]. Our direct inhibition study with CBD derivatives and molecular modeling revealed that the pentylresorcinol structure in $\mathrm{CBD}$ had structurally important roles in direct CYP1A1 
inhibition; however, the whole structure of CBD is known to be required for overall inhibition [13]. These studies on structure-inhibition relationships may provide an insight into the mechanism(s) underlying the potent inhibition of human CYP1A1 by CBD. However, the structural requirements for the potent mechanism-based inhibition of human CYP1A1 by CBD remain unclear.

In the present study, the inactivating effects of CBD-related compounds (Fig. 1) on human CYP1A1 activity were examined to elucidate the structural requirements for potent inactivation by $\mathrm{CBD}$. We report herein that the methylresorcinol structure in $\mathrm{CBD}$ may play important roles in CYP1A1 inactivation.

\section{Materials and methods}

\subsection{Materials}

CBD was isolated from cannabis leaves using a previously reported method [14]. The CBD-2 -monomethyl ether (CBDM) and CBD-2,6 -dimethyl ether (CBDD) were prepared as described previously [15]. CBD-hydroxyquinone (CBDHQ) was synthesized according to the method of Mechoulam et al. [16]. The purities of these cannabinoids were determined to be above $97 \%$ by gas chromatography, except for CBDD, the purity of which was $93 \%$ [17]. Cannabidivarin (CBDV) was generously provided by Dr. Yukihiro Shoyama at Nagasaki International University (Sasebo, Japan). Other chemicals and materials were obtained from the following sources: microsomes from baculovirus-infected insect cells expressing CYP1A1 with NADPH-CYP reductase (Supersomes ${ }^{\mathrm{TM}}$ ) from BD Gentest (Woburn, MA); 
7-ethoxyresorufin, resorufin, olivetol, $d$-limonene, and pentylbenzene from Sigma-Aldrich Corp. (St. Louis, MO); NADPH from Oriental Yeast Co. Ltd. (Tokyo, Japan); resorcinol from Wako Pure Chemical Industries, Ltd. (Osaka, Japan); orcinol obtained from Tokyo Chemical Industry Co., Ltd. (Tokyo, Japan). All other chemicals and solvents used were of the highest quality commercially available.

\subsection{Inactivation studies}

Inhibition experiments were conducted using 96-well microtiter plates, as described below, to identify the potential metabolism-dependent inhibition of human CYP1A1 by CBD-related compounds. The preincubation mixture consisted of recombinant CYP1A1 (10 fmol), each CBD-related compound (up to $100 \mu \mathrm{M}$ ), $1.67 \mathrm{mM} \mathrm{NADPH}$, and $50 \mathrm{mM}$ Tris-HCl buffer ( $\mathrm{pH}$ 7.4) containing $1 \%$ fatty acid-free bovine serum albumin in a final volume of 180 $\mu 1$. After pre-warming at $37^{\circ} \mathrm{C}$ for $5 \mathrm{~min}$, the preincubation was initiated by the addition of NADPH. 7-ethoxyresorufin $O$-deethylase (EROD) reactions were initiated following a 20-min preincubation by adding $20 \mu \mathrm{l}$ of 7-ethoxyresorufin solution to the preincubation mixture (final substrate concentration, $150 \mathrm{nM}$ ), as described previously [5]. Fluorescence derived from resorufin formation was recorded every $5 \mathrm{~min}$ for $30 \mathrm{~min}$ using FLUOstar OPTIMA $^{\circledR}$ (BMG Labtech, Offenburg, Germany) with excitation and emission filters at 544 and $590 \mathrm{~nm}$, respectively. Co-incubations in which 7-ethoxyresorufin was added together with $\mathrm{NADPH}$ at the end of $5 \mathrm{~min}$ of pre-warming were also carried out. The $\mathrm{IC}_{50}$ value was calculated by nonlinear regression analysis with GraphPad Prism 5.02 (GraphPad Software 
Inc., San Diego, CA) using the dose-response with variable slope function.

The kinetics of CYP1A1 inactivation by CBD and its structurally related compounds were determined as described previously [5] with minor modifications. Briefly, all reactions were performed at $37^{\circ} \mathrm{C}$ in a shaking water bath. Preincubation tubes contained recombinant human CYP1A1 (50 fmol), each CBD-related compound (up to $100 \mu \mathrm{M}$ ), $1.67 \mathrm{mM}$ NADPH, and $50 \mathrm{mM}$ Tris- $\mathrm{HCl}$ buffer ( $\mathrm{pH}$ 7.4) containing $1 \%$ fatty acid-free bovine serum albumin in a final volume of $100 \mu \mathrm{l}$. The preincubation was performed at $37^{\circ} \mathrm{C}$ for various amounts of time up to $30 \mathrm{~min}$. An aliquot $(20 \mu \mathrm{l})$ of the preincubation mixture was transferred to a $180 \mu \mathrm{l}$ enzyme assay mixture containing $150 \mathrm{nM}$ 7-ethoxyresorufin and $1.67 \mathrm{mM} \mathrm{NADPH}$, and the reaction was initiated. The reaction was terminated after a 20 -min incubation by the addition of $200 \mu \mathrm{l}$ of ice-cold methanol. NADPH was added following the termination of the reaction to obtain a background sample. After being kept on ice for $15 \mathrm{~min}, 300 \mu \mathrm{l}$ of the reaction mixture was transferred to a 96-well microtiter plate. Fluorescence was measured using FLUOstar OPTIMA $^{\circledR}$ (BMG Labtech) with the same filters as described above. The observed rates of CYP1A1 inactivation $\left(k_{\mathrm{obs}}\right)$ were calculated from the initial slopes of the linear regression lines of semilogarithmic plots (remaining EROD activity versus preincubation time). The obtained $k_{\text {obs }}$ were plotted against the cannabinoid concentration. The maximal inactivation constant $\left(k_{\text {inact }}\right)$ and half-maximal inhibitory concentration $\left(K_{\mathrm{I}}\right)$ were calculated by nonlinear regression analysis using GraphPad Prism 5.02 (GraphPad Software Inc.).

\subsection{Direct inhibition studies}


The effects of CBDHQ on CYP1A1 activity were determined using a 96-well microtiter plate as reported previously [5]. The apparent $K_{\mathrm{i}}$ value and mode of inhibition were determined by nonlinear regression analysis with GraphPad Prism 5.02 (GraphPad Software Inc.). Akaike's information criterion was used as a measure of goodness of fit. The mode of inhibition was verified by the visual inspection of Lineweaver-Burk plots of the enzyme kinetic data.

\section{Results}

3.1 Metabolism-dependent inhibition of human CYP1A1 by compounds structurally related to $C B D$

The effect of the preincubation on the extent of inhibition achieved by CBD-related compounds was investigated to determine whether these compounds inhibited CYP1A1-mediated EROD activity in a metabolism-dependent manner. A 20-min preincubation of $\mathrm{CBD}$ in the presence of NADPH strongly enhanced the inhibition of CYP1A1 activity (Fig. 2), as reported previously [5]. The preincubation of olivetol, which corresponds to the pentylresorcinol moiety of $\mathrm{CBD}$, potentiated the inhibition of CYP1A1 activity. In contrast, no increase was observed in inhibition following the preincubation of $d$-limonene, which corresponds to the terpene moiety of CBD (Fig. 2). Pentylbenzene, which lacks the two free phenolic hydroxyl groups in olivetol, did not potentiate CYP1A1 inhibition (Fig. 2). On the other hand, the inhibition of CYP1A1 activity by the monomethylated and dimethylated derivatives of CBD, CBDM and CBDD, respectively, was augmented by their 
preincubation (Fig. 2). The preincubation of CBDV, in which the pentyl side chain of CBD was substituted with a propyl side chain, strongly enhanced CYP1A1 inhibition (Fig. 2). Orcinol, which possesses a methyl group instead of the pentyl group in olivetol, potentiated the inhibition of CYP1A1 activity (Fig. 2). In contrast, resorcinol, which lacks the alkyl side chain of olivetol, did not augment CYP1A1 inhibition (Fig. 2). These results indicated that olivetol, CBDM, CBDD, CBDV, and orcinol as well as CBD inhibited CYP1A1 activity in a metabolism-dependent manner, as shown in Table 1.

\subsection{Inactivation of human CYP1A1 activity by compounds structurally related to $C B D$}

To characterize the parameters necessary to inactivate human CYP1A1 by CBD-related compounds, enzyme kinetics were analyzed under the six incubation conditions in which $\mathrm{IC}_{50}$ values were decreased due to the preincubation, as shown in Figure 2. In addition, a similar experiment was performed with resorcinol, which did not show any metabolism-dependent inhibition. The preincubation of CBD in the presence of NADPH caused a time- and concentration-dependent decrease in CYP1A1-mediated EROD activity (Fig. 3), as reported previously [5]. CYP1A1 inhibition by all the CBD-related compounds tested, except for resorcinol, was also preincubation time- and concentration-dependent (Fig. 3). As shown in Table 2 , the $k_{\text {inact }}$ value of olivetol was similar to that of CBD; however, olivetol showed a 10 -fold higher $K_{\mathrm{I}}$ value than that of CBD. The $K_{\mathrm{I}}$ values of CBDM and CBDD were 3- and 14-fold higher, respectively, than that of CBD (Table 2) and increased as the number of free phenolic hydroxyl groups decreased. Although the $k_{\text {inact }}$ values of CBDM 
and CBDD were equivalent, these values were approximately one-third that of CBD (Table 2). The $K_{\mathrm{I}}$ value of CBDV was slightly higher than that of CBD, but was similar to that of CBD (Table 2). The $K_{\mathrm{I}}$ and $k_{\text {inact }}$ values of orcinol were 193-fold higher and 6-fold lower, respectively, than those of CBD (Table 2).

\subsection{Effects of CBDHQ on human CYP1A1 activity}

Bornheim and Grillo [18] previously reported that CBDHQ, a hydroxyquinone metabolite produced from CBD by mouse CYP3A11, was a reactive intermediate that could inactivate CYP3A11. Thus, the effects of CBDHQ on human CYP1A1 activity were investigated to determine whether CBDHQ was a reactive metabolite that could inactivate CYP1A1. The results of the direct inhibition study revealed that CBDHQ competitively inhibited CYP1A1-mediated EROD activity (Fig. 4A). The $K_{\mathrm{i}}$ value of CBDHQ was $11.2 \mu \mathrm{M}$, which was 72-fold higher than that of $\operatorname{CBD}\left(K_{\mathrm{i}}=0.155 \mu \mathrm{M}\right)$ [5]. The effects of preincubation on CYP1A1 inhibition demonstrated that CBDHQ did not markedly (or significantly) potentiate the inhibition of CYP1A1 activity (Fig. 4B); $\mathrm{IC}_{50}$ values for the 0 - and 20-min preincubation of CBDHQ were 48.0 and $37.7 \mu \mathrm{M}$, respectively.

\section{Discussion}

Although previous studies reported that CBD inactivated the catalytic activities of human CYP1A1, CYP1A2, CYP1B1, and CYP3A [5, 19], a kinetic analysis has not yet been conducted for CYP3A inactivation. The potency of the CBD-mediated inactivation of 
CYP1A1 was shown to be at least 15-fold higher than those of CYP1A2 and CYP1B1, as assessed by the $k_{\text {inact }} / K_{\mathrm{I}}$ value [5]. On the other hand, CBD was shown to be a metabolism-dependent, but not mechanism-based inhibitor for CYP2A6 [6]. Furthermore, CBD did not inhibit CYP2B6 [6], CYP2C9 [9], CYP2C19 (unpublished data), or CYP2D6 in a metabolism-dependent manner [8]. These findings indicated that CBD was the most potent inactivator of human CYP1A1 among the CYP enzymes examined so far.

In the present study, we investigated the inhibiting effects of nine CBD-related compounds on CYP1A1 activity to clarify the structural requirements for potent mechanism-based inhibition by CBD. The mechanism-based inhibition of CYP1A1 by olivetol, but not $d$-limonene suggests that the pentylresorcinol structure in CBD may play an important role in this inactivation. The lack of both free phenolic hydroxyl groups in olivetol (i.e. pentylbenzene) abolished the metabolism-dependent inhibition of CYP1A1. Furthermore, inactivation studies on $\mathrm{CBDM}$ and $\mathrm{CBDD}$ revealed that the methylation of either phenolic hydroxyl group in CBD led to a marked decrease in $k_{\text {inact }}$ values. A hydroxyl group at the 2 -position in $\mathrm{CBD}$ was equivalent to the hydroxyl group at the 6 -position because of the free rotation of the phenyl ring around the chemical bond at the 1 -position (Fig. 1). These results suggest that both free phenolic hydroxyl groups in CBD may be required for potent inactivation. The substitution of the pentyl group of CBD with a propyl group (i.e. CBDV) did not influence the inhibition of CYP1A1 activity, which implies that carbon atoms at the 4"- and 5"-positions of the pentyl group in CBD were not involved in the inactivation of CYP1A1. The ability to inhibit CYP1A1 activity, even if the side chain in the pentylresorcinol 
structure was shortened to a methyl group, was preserved to some extent in orcinol. However, deletion of the side chain (i.e. resorcinol) caused a loss in the ability to inhibit CYP1A1 activity. These results suggest that at least the carbon atom at the 1 "-position of the pentyl group in CBD may be required for CYP1A1 inactivation. The results described above suggest that the methylresorcinol structure in CBD may play a key role in CYP1A1 inactivation. One possibility is that the formation of an electrophilic quinone from the methylresorcinol moiety of CBD by human CYP1A1 may lead to CBD-mediated inactivation. However, there is currently no direct evidence that this is involved in the inactivation of CYP1A1.

A previous study reported that CBD inactivated CYP3A-mediated oxidation in human and mouse liver microsomes [19]. CBDHQ may be a reactive metabolite that is produced from CBD by mouse CYP3A11, which ultimately inactivates this enzyme [18]. CBDHQ that formed during the incubation of CBD with mouse CYP3A11 was shown to be trapped as a glutathione adduct [18]. However, we previously demonstrated that glutathione as well as $\mathrm{N}$-acetylcysteine did not suppress the inactivation of human CYP1A1 by CBD [5]. Furthermore, superoxide dismutase, a scavenger of reactive oxygen species, did not protect human CYP1A1 from CBD-mediated inactivation. In the present study, the ability of CBDHQ to inhibit human CYP1A1 activity was markedly lower than that of CBD. Therefore, CBDHQ is unlikely to be the reactive intermediate causing CYP1A1 inactivation. However, the reactive intermediate leading to the mechanism-based inhibition of human CYP1A1 by CBD has not yet been identified even though the structure of CBD required for this inactivation is known. 
Although CYP1A1 is expressed in various tissues including the liver and lung [20-22], its constitutive expression level is very low. Hepatic and pulmonary CYP1A1 is generally induced following exposure to tobacco smoke $[23,24]$. CYP1A1 is known to play a critical role in the metabolic activation of procarcinogens, such as polycyclic aromatic hydrocarbons and heterocyclic amines $[22,25]$. The bioactivation of these procarcinogens may induce genotoxicity and carcinogenicity [26]. Therefore, (phyto)chemicals that potently and selectively inhibit CYP1A1 activity are expected to be lead compounds in anticancer chemotherapy. Once a mechanism-based inhibitor inactivates a target enzyme, its inhibitory effect persists until the enzyme is newly synthesized. Thus, it is possible that a mechanism-based inhibitor for CYP1A1 has a higher anti-tumorigenic effect than that of a direct inhibitor for CYP1A1. Although many direct CYP1A1 inhibitors have been identified, there are only a limited number of metabolism-dependent inhibitors and inactivators for human CYP1A1 (Table 3). Rhapontigenin, a stilbene derivative isolated from the rhizome of Rheum undulatum, may be a promising anticancer drug because this phytochemical has been shown to potently inactivate human CYP1A1 activity [27]. The potency of the CBD-mediated inactivation of CYP1A1 was similar to that of rhapontigenin, as assessed by $k_{\text {inact }} / K_{\mathrm{I}}$ values (Table 3). Based on these findings, CBD may also be a potential lead compound in chemotherapy for the treatment of cancer, especially lung cancer.

\section{Conclusions}

We demonstrated that several compounds structurally related to CBD as well as CBD 
inhibited human CYP1A1 activity. Our results suggest that the methylresorcinol structure in CBD may be required for CYP1A1 inactivation. This study has provided useful information that will lead to a clearer understanding of the precise mechanism(s) underlying the potent CBD inactivation of human CYP1A1. 


\section{Acknowledgements}

This work was supported in part by a Grant-in-Aid for Young Scientists (B) and Grant-in-Aid for Scientific Research (C) from the Ministry of Education, Culture, Sports, Science, and Technology of Japan and by the 'Academic Frontier' Project for Private Universities from the Ministry of Education, Culture, Sports, Science, and Technology of Japan (2005-2009). The authors thank Dr. Yukihiro Shoyama (Faculty of Pharmaceutical Sciences, Nagasaki International University, Sasebo, Japan) for generously providing CBDV. 


\section{Conflict of interest statement}

The authors declare that there are no conflicts of interest. 


\section{References}

[1] M.A. ElSohly, D. Slade, Chemical constituents of marijuana: the complex mixture of natural cannabinoids, Life Sci. 78 (2005) 539-548.

[2] R. Mechoulam, L.A. Parker, R. Gallily, Cannabidiol: an overview of some pharmacological aspects, J. Clin. Pharmacol. 42 (2002) 11S-19S.

[3] P. Massi, M. Solinas, V. Cinquina, D. Parolaro, Cannabidiol as potential anticancer drug, Br. J. Clin. Pharmacol. 75 (2013) 303-312.

[4] N.L. Benowitz, T.L. Nguyen, R.T. Jones, R.I. Herning, J. Bachman, Metabolic and psychophysiologic studies of cannabidiol-hexobarbital interaction, Clin. Pharmacol. Ther. 28 (1980) 115-120.

[5] S. Yamaori, M. Kushihara, I. Yamamoto, K. Watanabe, Characterization of major phytocannabinoids, cannabidiol and cannabinol, as isoform-selective and potent inhibitors of human CYP1 enzymes, Biochem. Pharmacol. 79 (2010) 1691-1698.

[6] S. Yamaori, C. Maeda, I. Yamamoto, K. Watanabe, Differential inhibition of human cytochrome P450 2A6 and 2B6 by major phytocannabinoids, Forensic Toxicol. 29 (2011) 117-124.

[7] S. Yamaori, J. Ebisawa, Y. Okushima, I. Yamamoto, K. Watanabe, Potent inhibition of human cytochrome P450 3A isoforms by cannabidiol: role of phenolic hydroxyl groups in the resorcinol moiety, Life Sci. 88 (2011) 730-736.

[8] S. Yamaori, Y. Okamoto, I. Yamamoto, K. Watanabe, Cannabidiol, a major phytocannabinoid, as a potent atypical inhibitor for CYP2D6, Drug Metab. Dispos. 39 
(2011) 2049-2056.

[9] S. Yamaori, K. Koeda, M. Kushihara, Y. Hada, I. Yamamoto, K. Watanabe, Comparison in the in vitro inhibitory effects of major phytocannabinoids and polycyclic aromatic hydrocarbons contained in marijuana smoke on cytochrome P450 2C9 activity, Drug Metab. Pharmacokinet. 27 (2012) 294-300.

[10] R. Jiang, S. Yamaori, Y. Okamoto, I. Yamamoto, K. Watanabe, Cannabidiol is a potent inhibitor of the catalytic activity of cytochrome P450 2C19, Drug Metab. Pharmacokinet. 28 (2013) 332-338.

[11] S. Takeda, K. Misawa, I. Yamamoto, K. Watanabe, Cannabidiolic acid as a selective cyclooxygenase-2 inhibitory component in cannabis, Drug Metab. Dispos. 36 (2008) 1917-1921.

[12] S. Takeda, N. Usami, I. Yamamoto, K. Watanabe, Cannabidiol-2,6 -dimethyl ether, a cannabidiol derivative, is a highly potent and selective 15-lipoxygenase inhibitor, Drug Metab. Dispos. 37 (2009) 1733-1737.

[13] S. Yamaori, Y. Okushima, K. Masuda, M. Kushihara, T. Katsu, S. Narimatsu, I. Yamamoto, K. Watanabe, Structural requirements for potent direct inhibition of human cytochrome P450 1A1 by cannabidiol: role of pentylresorcinol moiety, Biol. Pharm. Bull. 36 (2013) 1197-1203.

[14] H. Aramaki, N. Tomiyasu, H. Yoshimura, H. Tsukamoto, Forensic chemical study on marihuana. I. A detection method of the principal constituents by thin-layer and gas chromatographies, Chem. Pharm. Bull. 16 (1968) 822-826. 
[15] H. Gohda, S. Narimatsu, I. Yamamoto, H. Yoshimura, In vivo and in vitro metabolism of cannabidiol monomethyl ether and cannabidiol dimethyl ether in the guinea pig: on the formation mechanism of cannabielsoin-type metabolite from cannabidiol, Chem. Pharm. Bull. 38 (1990) 1697-1701.

[16] R. Mechoulam, Z. Ben-Zvi, Y. Gaoni, Hashish--13. On the nature of the Beam test, Tetrahedron 24 (1968) 5615-5624.

[17] K. Watanabe, E. Motoya, N. Matsuzawa, T. Funahashi, T. Kimura, T. Matsunaga, K. Arizono, I. Yamamoto, Marijuana extracts possess the effects like the endocrine disrupting chemicals, Toxicology 206 (2005) 471-478.

[18] L.M. Bornheim, M.P. Grillo, Characterization of cytochrome P450 3A inactivation by cannabidiol: possible involvement of cannabidiol-hydroxyquinone as a P450 inactivator, Chem. Res. Toxicol. 11 (1998) 1209-1216.

[19] W. Jaeger, L.Z. Benet, L.M. Bornheim, Inhibition of cyclosporine and tetrahydrocannabinol metabolism by cannabidiol in mouse and human microsomes, Xenobiotica 26 (1996) 275-284.

[20] S.A. Wrighton, C. Campanile, P.E. Thomas, S.L. Maines, P.B. Watkins, G. Parker, G. Mendez-Picon, M. Haniu, J.E. Shively, W. Levin, Identification of a human liver cytochrome P-450 homologous to the major isosafrole-inducible cytochrome P-450 in the rat, Mol. Pharmacol. 29 (1986) 405-410.

[21] T. Shimada, C.H. Yun, H. Yamazaki, J.C. Gautier, P.H. Beaune, F.P. Guengerich, Characterization of human lung microsomal cytochrome P-450 1A1 and its role in the 
oxidation of chemical carcinogens, Mol. Pharmacol. 41 (1992) 856-864.

[22] T. Shimada, C.L. Hayes, H. Yamazaki, S. Amin, S.S. Hecht, F.P. Guengerich, T.R. Sutter, Activation of chemically diverse procarcinogens by human cytochrome P-450 1B1, Cancer Res. 56 (1996) 2979-2984.

[23] J.C. Willey, E.L. Coy, M.W. Frampton, A. Torres, M.J. Apostolakos, G. Hoehn, W.H. Schuermann, W.G. Thilly, D.E. Olson, J.R. Hammersley, C.L. Crespi, M.J. Utell, Quantitative RT-PCR measurement of cytochromes p450 1A1, 1B1, and 2B7, microsomal epoxide hydrolase, and NADPH oxidoreductase expression in lung cells of smokers and nonsmokers, Am. J. Respir. Cell Mol. Biol. 17 (1997) 114-124.

[24] J.H. Kim, M.E. Sherman, F.C. Curriero, F.P. Guengerich, P.T. Strickland, T.R. Sutter, Expression of cytochromes P450 1A1 and 1B1 in human lung from smokers, non-smokers, and ex-smokers, Toxicol. Appl. Pharmacol. 199 (2004) 210-219.

[25] T. Shimada, Y. Fujii-Kuriyama, Metabolic activation of polycyclic aromatic hydrocarbons to carcinogens by cytochromes P450 1A1 and 1B1, Cancer Sci. 95 (2004) 1-6.

[26] T. Shimada, Xenobiotic-metabolizing enzymes involved in activation and detoxification of carcinogenic polycyclic aromatic hydrocarbons, Drug Metab. Pharmacokinet. 21 (2006) 257-276.

[27] Y.J. Chun, S.Y. Ryu, T.C. Jeong, M.Y. Kim, Mechanism-based inhibition of human cytochrome P450 1A1 by rhapontigenin, Drug Metab. Dispos. 29 (2001) 389-393.

[28] T. Shimada, N. Murayama, K. Okada, Y. Funae, H. Yamazaki, F.P. Guengerich, 
Different mechanisms for inhibition of human cytochromes P450 1A1, 1A2, and 1B1 by polycyclic aromatic inhibitors, Chem. Res. Toxicol. 20 (2007) 489-496.

[29] Y.F. Ueng, C.C. Chen, H. Yamazaki, K. Kiyotani, Y.P. Chang, W.S. Lo, D.T. Li, P.L. Tsai, Mechanism-based inhibition of CYP1A1 and CYP3A4 by the furanocoumarin chalepensin, Drug Metab. Pharmacokinet. 28 (2013) 229-238.

[30] J. Sridhar, J. Ellis, P. Dupart, J. Liu, C.L. Stevens, M. Foroozesh, Development of flavone propargyl ethers as potent and selective inhibitors of cytochrome P450 enzymes 1A1 and 1A2, Drug Metab. Lett. 6 (2012) 275-284.

[31] J. Sridhar, J. Liu, M. Foroozesh, C.L. Klein Stevens, Inhibition of cytochrome p450 enzymes by quinones and anthraquinones, Chem. Res. Toxicol. 25 (2012) 357-365.

[32] M. Nakajima, M. Suzuki, R. Yamaji, H. Takashina, N. Shimada, H. Yamazaki, T. Yokoi, Isoform selective inhibition and inactivation of human cytochrome P450s by methylenedioxyphenyl compounds, Xenobiotica 29 (1999) 1191-1202.

[33] K. Ohyama, M. Nakajima, M. Suzuki, N. Shimada, H. Yamazaki, T. Yokoi, Inhibitory effects of amiodarone and its N-deethylated metabolite on human cytochrome P450 activities: prediction of in vivo drug interactions, Br. J. Clin. Pharmacol. 49 (2000) 244-253. 


\section{Footnotes}

${ }^{1}$ Present address: Department of Pharmacy, Shinshu University Hospital, 3-1-1 Asahi, Matsumoto 390-8621, Japan. 


\section{Table 1}

Summary of the $\mathrm{IC}_{50}$ values of $\mathrm{CBD}$ and its structurally related compounds for CYP1A1 inhibition with or without preincubation.

\begin{tabular}{lccl}
\hline \multirow{2}{*}{ Compounds } & \multicolumn{2}{c}{$\mathrm{IC}_{50}(\mu \mathrm{M})$} & \\
\cline { 2 - 3 } & \multicolumn{2}{c}{ Preincubation time } & \\
& 0 min $(\mathrm{A})$ & $20 \min (\mathrm{B})$ & $\mathrm{B} / \mathrm{A}$ \\
\hline CBD & 0.671 & 0.0678 & 0.101 \\
Olivetol & 11.1 & 2.60 & 0.234 \\
$d$-Limonene & $>100$ & $>100$ & - \\
Pentylbenzene & $>100$ & $>100$ & - \\
CBDM & 4.12 & 1.90 & 0.461 \\
CBDD & 29.2 & 7.68 & 0.263 \\
CBDV & 1.64 & 0.0677 & 0.0413 \\
Orcinol & 62.3 & 38.7 & 0.621 \\
Resorcinol & $>100$ & $>100$ & - \\
\hline
\end{tabular}

All determinations were performed in duplicate. 


\section{Table 2}

Kinetic parameters for the mechanism-based inhibition of human CYP1A1 by CBD and its structurally related compounds.

\begin{tabular}{lccc}
\hline Compounds & $k_{\text {inact }}\left(\mathrm{min}^{-1}\right)$ & $K_{\mathrm{I}}(\mu \mathrm{M})$ & $k_{\text {inact }} / K_{\mathrm{I}}\left(1 \mathrm{mmol}^{-1} \mathrm{~min}^{-1}\right)$ \\
\hline CBD & 0.215 & 0.439 & 490 \\
Olivetol & 0.154 & 4.66 & 33.0 \\
CBDM & 0.0638 & 1.33 & 48.0 \\
CBDD & 0.0643 & 6.11 & 10.5 \\
CBDV & 0.226 & 0.623 & 363 \\
Orcinol & 0.0353 & 84.7 & 0.417 \\
\hline
\end{tabular}




\section{Table 3}

Summary of the kinetic parameters for the mechanism-based inhibitors of human CYP1A1.

\begin{tabular}{|c|c|c|c|c|}
\hline Inactivators & $\begin{array}{c}k_{\text {inact }} \\
\left(\min ^{-1}\right)\end{array}$ & $\begin{array}{c}K_{\mathrm{I}} \\
(\mu \mathrm{M})\end{array}$ & $\begin{array}{c}k_{\text {inact }} / K_{\mathrm{I}} \\
\left(1 \mathrm{mmol}^{-1} \mathrm{~min}^{-1}\right)\end{array}$ & Refs. \\
\hline 1-Ethynylpyrene & 0.24 & 0.16 & 1500 & {$[28]$} \\
\hline 1-(1-Propynyl)pyrene & 0.24 & 0.16 & 1500 & {$[28]$} \\
\hline Rhapontigenin & 0.06 & 0.09 & 700 & {$[27]$} \\
\hline Chalepensin & 0.400 & 0.64 & 625 & [29] \\
\hline 3 -Flavonepropargylether & 0.090 & 0.24 & 380 & {$[30]$} \\
\hline Emodin compound $1^{\mathrm{a}}$ & 1.57 & 5.38 & 292 & {$[31]$} \\
\hline 4-(1-Propynyl)biphenyl & 0.11 & 1.5 & 73 & {$[28]$} \\
\hline 7-Hydroxyflavone & 0.115 & 2.43 & 47 & {$[30]$} \\
\hline Methylenedioxyphenyl compound ${ }^{\mathrm{b}}$ & 0.034 & 0.81 & 42 & {$[32]$} \\
\hline Desethylamiodarone & 0.03 & 1.0 & 30 & {$[33]$} \\
\hline
\end{tabular}

a 1-Amino-4-chloro-2-methylanthracene-9,10-dione.

b 2-[5-Methoxy-2-[5-methyl[2-(3,4-methylenedioxyphenoxy)ethyl]amino]pentyloxy]phenyl]4-methyl-3-oxo-2H-1,4-benzothiazine. 


\section{Figure legends}

Fig. 1. Chemical structures of CBD and its structurally related compounds.

Fig. 2. Effect of preincubation on the inhibition of human CYP1A1 by CBD and its structurally related compounds. Recombinant human CYP1A1 was preincubated with CBD-related compounds ( 0 to $100 \mu \mathrm{M})$ in the presence of NADPH for 0 or $20 \mathrm{~min}$. EROD activity was measured after the addition of 7-ethoxyresorufin. Each point is the mean of duplicate determinations.

Fig. 3. Inactivation of human CYP1A1 by $\mathrm{CBD}$ and its structurally related compounds. Recombinant human CYP1A1 was preincubated with CBD-related compounds (0 to $100 \mu \mathrm{M})$ in the presence of NADPH for up to $30 \mathrm{~min}$. Aliquots were removed from the preincubation mixtures at the indicated time points and diluted 10-fold to measure residual activity. In the left graph, each point is the mean of duplicate determinations. The right graph shows the plot of $k_{\text {obs }}$ against CBD-related compounds.

Fig. 4. Effects of CBDHQ on human CYP1A1 activity. (A) Recombinant human CYP1A1 was incubated with 7-ethoxyresorufin in the presence of various amounts of CBDHQ. S and v indicate the concentration of 7-ethoxyresorufin (nM) and EROD activity (nmol min ${ }^{-1} \mathrm{nmol}^{-1}$ P450), respectively. (B) Recombinant human CYP1A1 was preincubated with CBDHQ (0 to $50 \mu \mathrm{M})$ in the presence of NADPH for 0 or 20 min. EROD activity was measured after the 
addition of 7-ethoxyresorufin. Each point is the mean of duplicate determinations. 


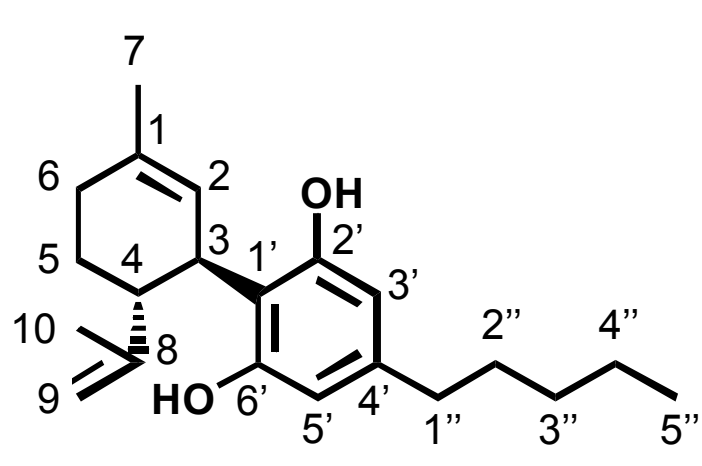

CBD

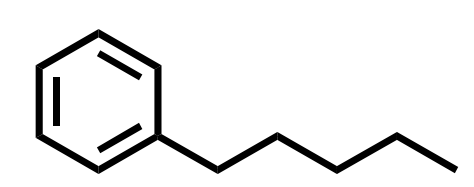

Pentylbenzene

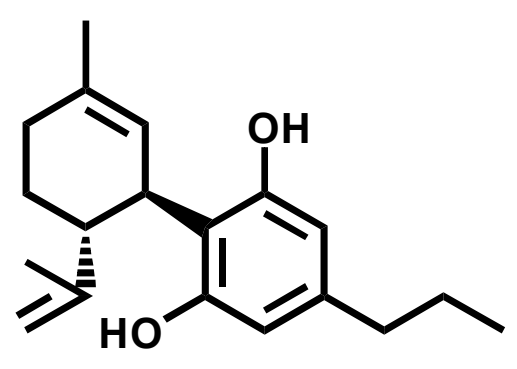

CBDV

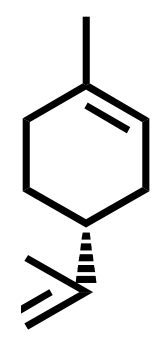

$d$-Limonene

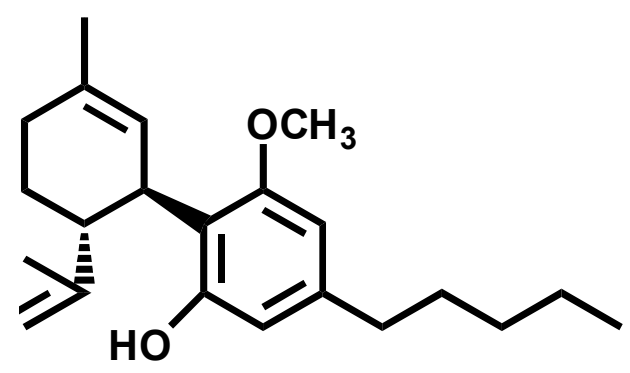

CBDM

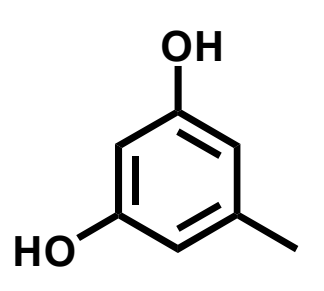

Orcinol<smiles>Oc1cccc(O)c1</smiles>

Resorcinol

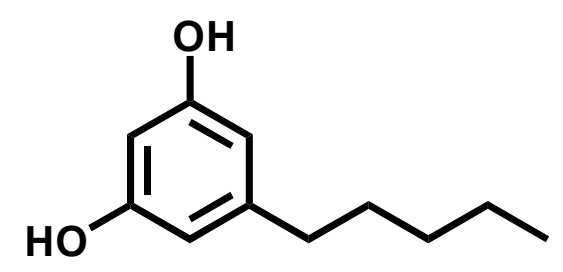

Olivetol

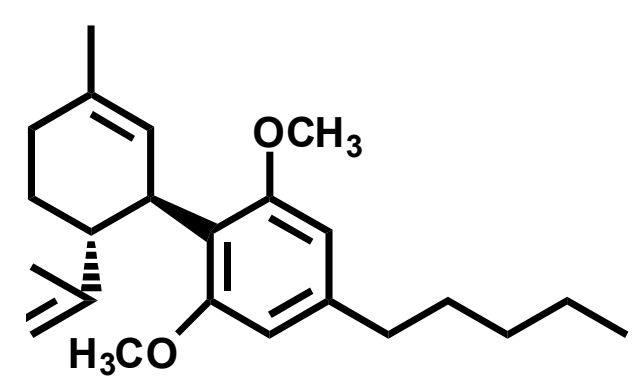

CBDD

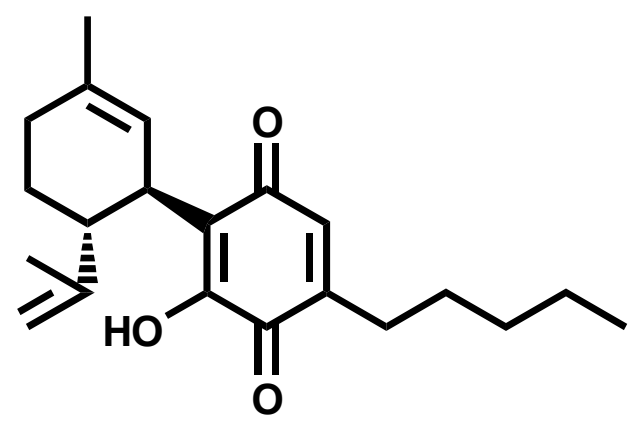

CBDHQ 

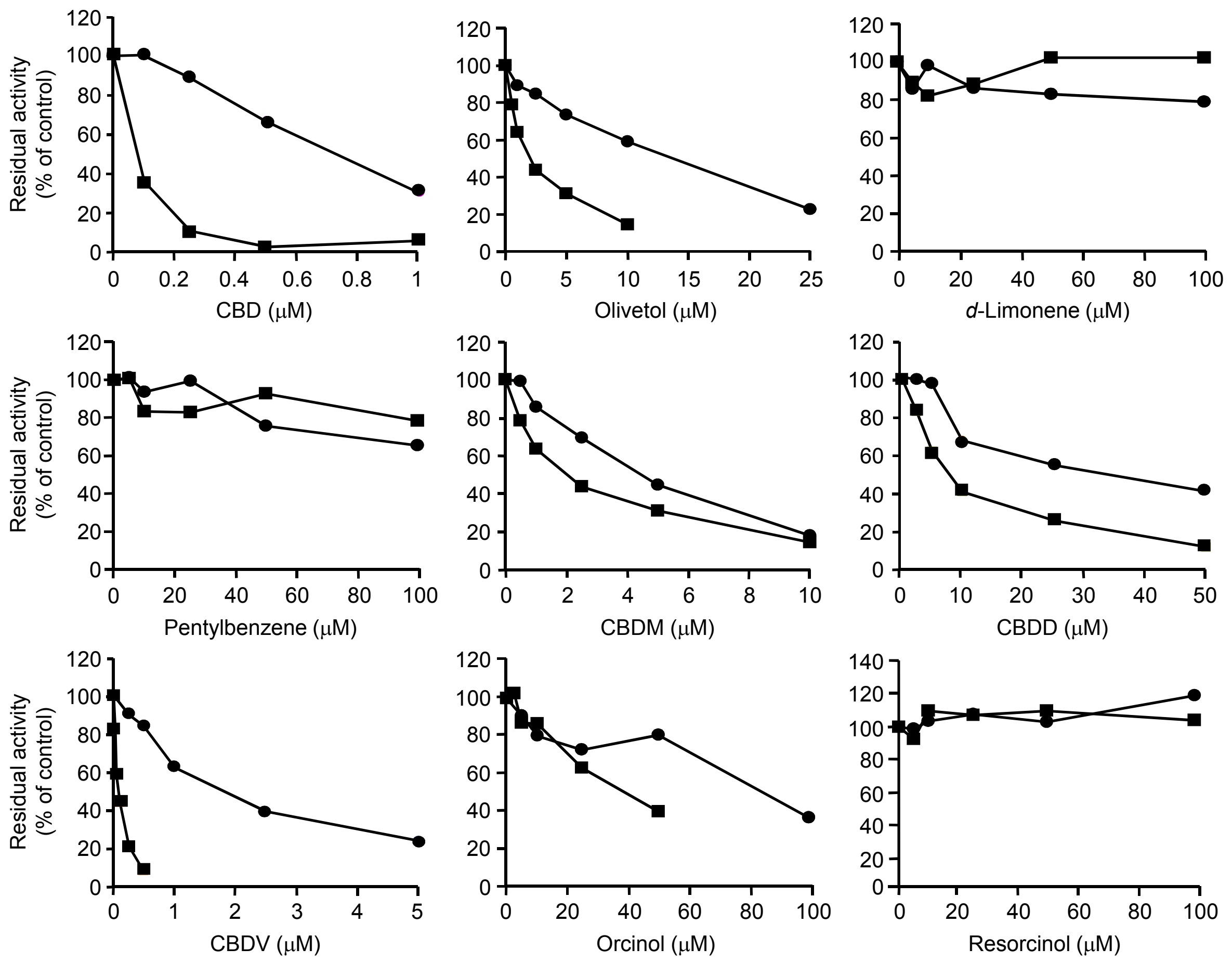

Fig. 2 

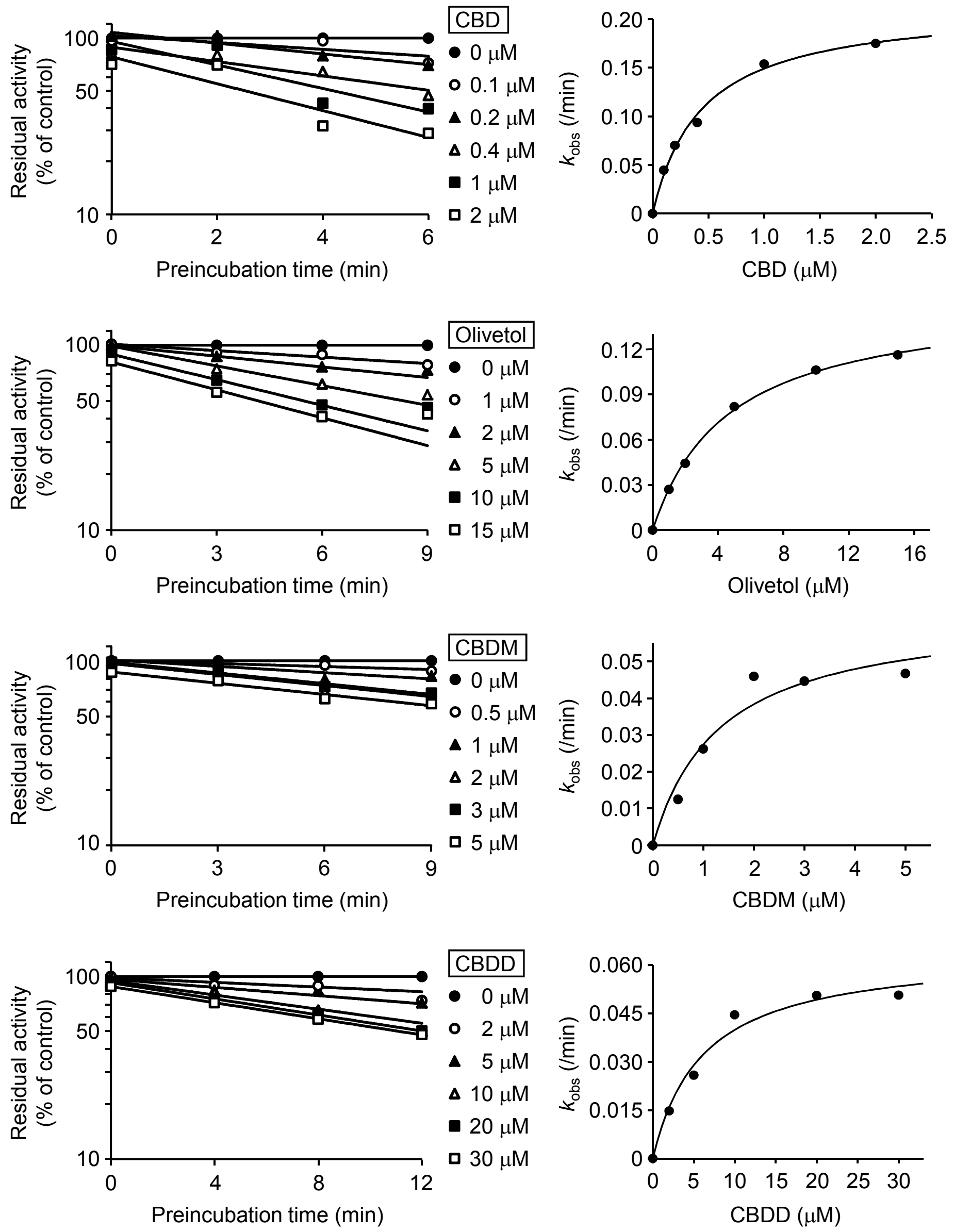

Fig. 3 

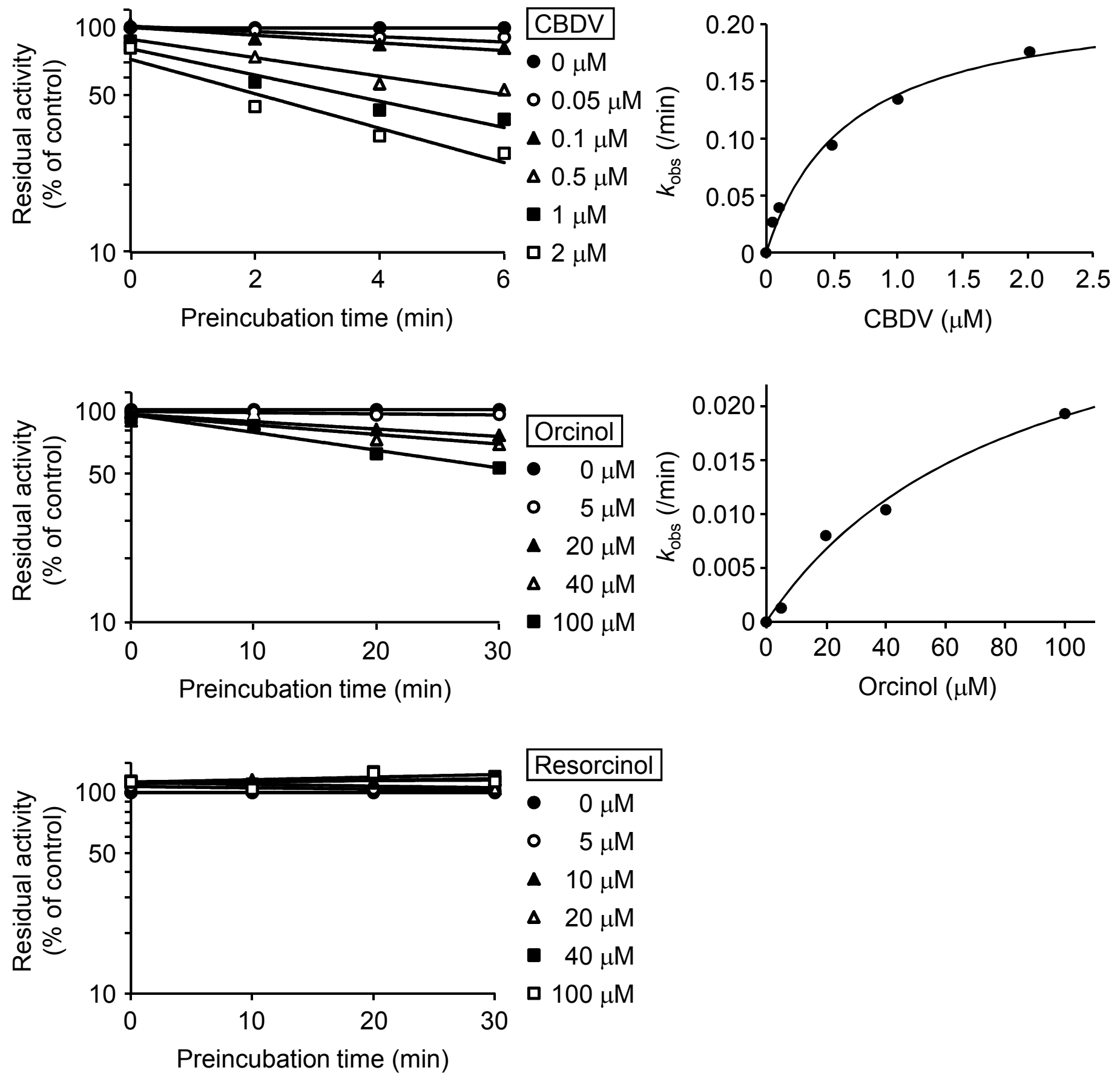
Resorcinol
- $0 \mu \mathrm{M}$
- $5 \mu \mathrm{M}$
- $10 \mu \mathrm{M}$
$\Delta 20 \mu \mathrm{M}$
- $40 \mu \mathrm{M}$
口 $100 \mu \mathrm{M}$

Fig. 3 (continued) 
(A)

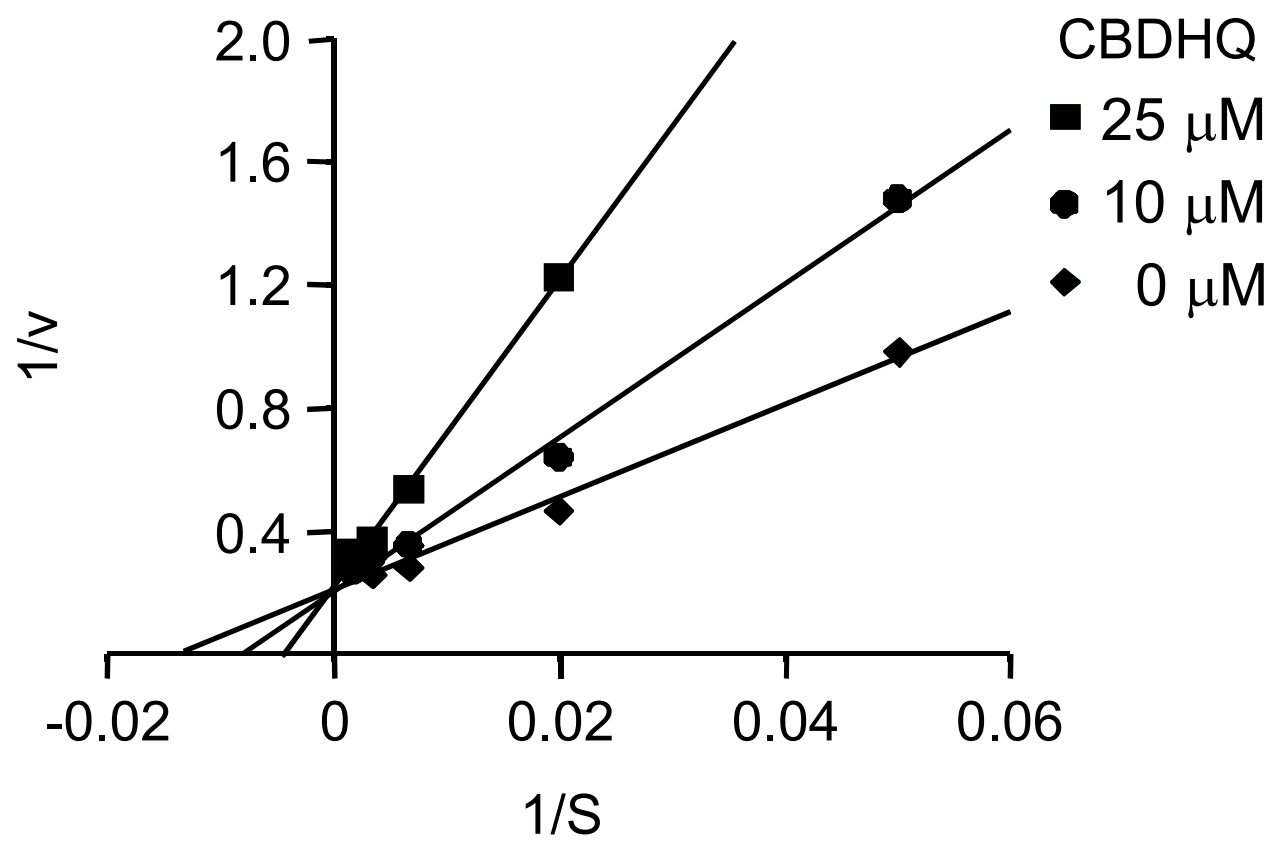

(B)

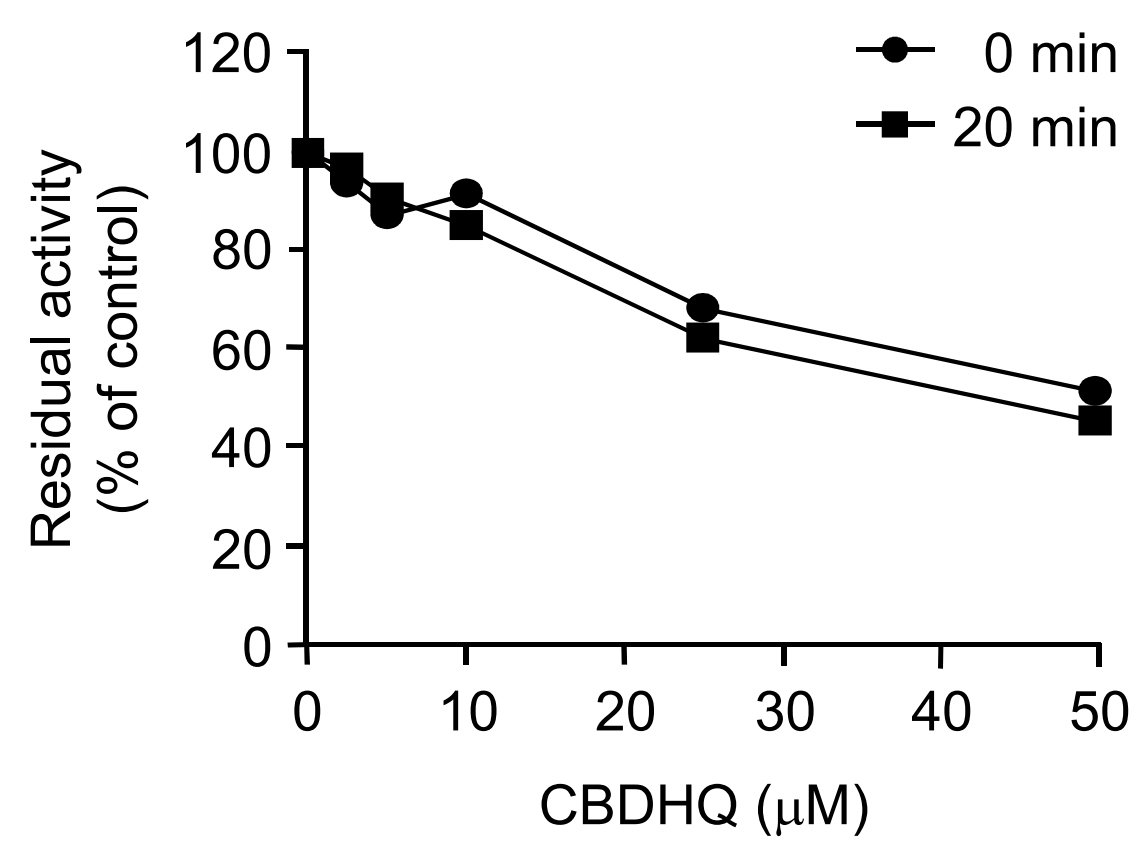

